\title{
Nicotinamide riboside regulates inflammation and mitochondrial markers in AML12 hepatocytes
}

\author{
Hee Jae Lee and Soo Jin Yang ${ }^{\S}$ \\ Department of Food and Nutrition, Seoul Women's University, 621 Hwarangro, Nowon-Gu, Seoul 01797, Korea
}

BACKGROUND/OBJECTIVES: The NAD ${ }^{+}$precursor nicotinamide riboside (NR) is a type of vitamin $\mathrm{B}_{3}$ found in cow's milk and yeast-containing food products such as beer. Recent studies suggested that NR prevents hearing loss, high-fat diet-induced obesity, Alzheimer's disease, and mitochondrial myopathy. The objective of this study was to investigate the effects of NR on inflammation and mitochondrial biogenesis in AML12 mouse hepatocytes.

MATERIALS/METHODS: A subset of hepatocytes was treated with palmitic acid (PA; $250 \mu \mathrm{M})$ for $48 \mathrm{~h}$ to induce hepatocyte steatosis. The hepatocytes were treated with NR $(10 \mu \mathrm{M}$ and $10 \mathrm{mM})$ for $24 \mathrm{~h}$ with and without PA. The cell viability and the levels of sirtuins, inflammatory markers, and mitochondrial markers were analyzed.

RESULTS: Cytotoxicity of NR was examined by PrestoBlue assay. Exposure to NR had no effect on cell viability or morphology. Gene expression of sirtuin 1 (Sirt1) and Sirt3 was significantly upregulated by NR in PA-treated hepatocytes. However, Sirt1 activities were increased in hepatocytes treated with low-dose NR. Hepatic pro-inflammatory markers including tumor necrosis factor-alpha and interleukin- 6 were decreased in NR-treated cells. NR upregulated anti-inflammatory molecule adiponectin, and, tended to down-regulate hepatokine fetuin-A in PA-treated hepatocytes, suggesting its inverse regulation on these cytokines. NR increased levels of mitochondrial markers including peroxisome proliferator-activated receptor $\mathrm{y}$ coactivator-1a, carnitine palmitoyltransferase 1, uncoupling protein 2, transcription factor A, mitochondrial and mitochondrial DNA in PA-treated hepatocytes. CONCLUSIONS: These data demonstrated that NR attenuated hepatic inflammation and increased levels of mitochondrial markers in hepatocytes.

Nutrition Research and Practice 2019;13(1):3-10; https://doi.org/10.4162/nrp.2019.13.1.3; pISSN 1976-1457 elSSN 2005-6168

Keywords: Fatty liver, inflammation, mitochondria, niacin

\section{INTRODUCTION}

Nicotinamide riboside (NR) is a recently discovered vitamin $B_{3}$, which is mainly present in milk [1]. It mediates several physiological processes acting as an $\mathrm{NAD}^{+}$precursor [2,3]. NR is converted to $\mathrm{NAD}^{+}$via two pathways: NR kinase (NRK) pathway and nicotinamide (NAM) salvage pathway [2,3]. NR phosphorylation by NRK produces nicotinamide mononucleotide (NMN), which is adenylated to $\mathrm{NAD}^{+}$by nicotinamide mononucleotide adenylyltransferase [3]. NR may break down to NAM by purine nucleoside phosphorylase [2]. Nicotinamide phosphoribosyltransferase catalyzes NAM to NMN, which is metabolized to $\mathrm{NAD}^{+}$.

Nicotinic acid (NA), which is one version of niacin, is used to treat dyslipidemia, especially by increasing high-density lipoprotein cholesterol as well as by decreasing triglyceride and low-density lipoprotein cholesterol in humans [4]. However, high doses (more than $1 \mathrm{~g} /$ day) of NA often induce flushing response, which limits the use of NA as a pharmacological agent
[5]. NAM is a more effective $\mathrm{NAD}^{+}$precursor than other types of niacin in stressed conditions including exposure to high-fat (HF) diet and hyperglycemia [6]. It improves systemic glucose control and hepatic NAD ${ }^{+}$-sirtuin pathway as well as mitochondrial biogenesis [6]. NAM preserves the mass and function of pancreatic cells [7]. However, its potential side effects include liver toxicity, oncogenicity, and growth inhibition in animals and humans [8]. In contrast, no side effects of NR have yet to be reported. In addition, oral intake of NR elevates hepatic NAD more effectively in mouse compared with NA or NAM [9]. However, NR is more expensive than other types of niacin. Both in vitro and in vivo studies have demonstrated the potential of NR to improve hearing loss, obesity, and mitochondrial myopathy. The mechanism of action involves increased $\mathrm{NAD}^{+}$ levels, activation of sirtuins (Sirt) and induction of mitochondrial biogenesis [10-12]. NR supplementation prevented the development of diabetic neuropathy in a rodent model of type 2 diabetes [13].

$\mathrm{NR}$ elevates or maintains $\mathrm{NAD}^{+}$levels in liver, which may affect

\footnotetext{
This study was supported by a grant of the Basic Science Research Program through the National Research Foundation of Korea (NRF) funded by the Ministry of Education (NRF-2017R1D1A1B03030617). The funder had no role in study design, data collection and analysis, decision to publish, or preparation of the manuscript ${ }^{\S}$ Corresponding Author: Soo Jin Yang, Tel. 82-2-970-5643, Fax. 82-2-976-4049, Email. sjyang89@swu.ac.kr Received: April 24, 2018, Revised: June 6, 2018, Accepted: October 30, 2018

This is an Open Access article distributed under the terms of the Creative Commons Attribution Non-Commercial License (http://creativecommons.org/licenses/by-nc/3.0/) which permits unrestricted non-commercial use, distribution, and reproduction in any medium, provided the original work is properly cited.
} 
the development of non-alcoholic fatty liver disease (NAFLD) by altering biological pathways including mitochondrial unfolded protein responses and $\mathrm{NAD}^{+}$-dependent Sirt pathway $[10,14]$. Short-term NR treatment decreased hepatic nucleotide binding and oligomerization domain-like receptor family, pyrin domain containing 3 (NLRP3) inflammasome in obese/diabetic KK mice [15]. Increasing NAD ${ }^{+}$by NA supplementation ameliorated hepatic steatosis and increased hepatic biogenesis [16].

$\mathrm{NR}$ is available from commercial reagent suppliers, and an oral supplement was also introduced under the brand name NIAGEN ${ }^{\circledR}$. However, the evidence of health benefits, especially for liver, is still limited. Therefore, we hypothesized that NR ameliorates hepatic inflammation and increases levels of mitochondrial markers, and tested the hypothesis in an experimental model of hepatocyte steatosis.

\section{MATERIALS AND METHODS}

\section{Cell culture and treatments}

Mouse hepatocyte AML12 cells (American Type Culture Collection, Manassas, VA, USA) were cultured in DMEM/F12 medium (Invitrogen, Carlsbad, CA, USA) supplemented with $10 \%$ fetal bovine serum, penicillin-streptomycin $(100 \mathrm{U} / \mathrm{mL})$, a mixture of insulin, transferrin, and selenium and $0.1 \mathrm{mM}$ dexamethasone at $37^{\circ} \mathrm{C}$ in a humidified atmosphere enriched with $5 \% \mathrm{CO}_{2}$. Steatosis was induced by treating AML12 cells with $250 \mu \mathrm{M}$ palmitic acid (PA, Sigma-Aldrich, St. Louis, MO, USA) for $48 \mathrm{~h}$. The cells were treated with phosphate-buffered saline (PBS) or NR (10 $\mu \mathrm{M}$ and $10 \mathrm{mM}$; BOC Sciences, Shirley, NY, USA) for $24 \mathrm{~h}$. Morphological changes in the cells were captured using a Leica DM IL LED (Leica, Wetzlar, Germany) and TrueChrome Metrics (Fuzhou Tucsen Photonics Co., Fuzhou, Fujian, China). Original magnifications of acquired images were $100 \mathrm{x}$ and $200 \mathrm{x}$.

\section{Cell viability}

PrestoBlue ${ }^{\circledR}$ assay was performed according to the manufacturer's instructions. Each well contained the cells cultured medium removed. PrestoBlue ${ }^{\circledR}$ solution (Invitrogen) was added to each well, and the plates were incubated at $37^{\circ} \mathrm{C}$ with $5 \%$ $\mathrm{CO}_{2}$ for a specified duration. After incubation, the absorbance was measured using an Epoch microplate spectrophotometer (BioTek Instruments, Winooski, VT, USA) at $570 \mathrm{~nm}$.

\section{Oil Red O staining}

Cells were gently rinsed twice with PBS, fixed in a with $4 \%$ paraformaldehyde-PBS solution for $1 \mathrm{~h}$ at room temperature, stained with the $0.5 \%$ Oil Red O-isopropyl alcohol for $1 \mathrm{~h}$, and then washed with distilled water. The cells were checked by a Leica DM IL LED (Leica, Wetzlar, Germany) and TrueChrome Metrics (Fuzhou Tucsen Photonics Co., Fuzhou, Fujian, China). Original magnifications of acquired images were $40 \mathrm{x}$.

\section{Sirt1 activity}

Sirt1 activities in the cell were measured using a commercial Sirt1 activity assay kit (Abcam, Cambridge, UK), and the assay was conducted according to the manufacturer's instructions. $\mathrm{ddH}_{2} \mathrm{O}$, Sirt 1 assay buffer, fluoro-substrate peptide, NAD, and developer were mixed in microplate wells. Cell lysate was added to each well, and mixed at room temperature. Fluorescence intensity was read for $30 \mathrm{~min}$ to $1 \mathrm{~h}$ using SpectraMax M3 multiplate reader (Molecular Devices, Sunnyvale, CA, USA) with excitation/emission at 360/460 $\mathrm{nm}$.

RNA extraction and quantitative reverse transcriptase polymerase chain reaction ( $q R T-P C R$ ) analysis

Total RNA was isolated using PureLink RNA Mini kit (Invitrogen) according to the manufacturer's instructions. RNA concentration was measured on Nanodrop 2000 (Thermo Fisher Scientific, Wilmington, DE, USA). Reverse transcription was performed using High Capacity cDNA Reverse Transcription kit (Invitrogen). Power SYBR Green PCR Master Mix (Applied Biosystems, Forester city, CA, USA), CDNA and specific primers (Bioneer, Daejeon, Korea) were mixed. Real-time PCR was performed using StepOnePlus (Life Technologies, Carlsbad, CA, USA). Relative expression levels were determined based on the $\mathrm{Ct}$ values and normalized to $\mathrm{Ct}$ values for the $18 \mathrm{~S}$ gene.

\section{Western blot analysis}

Cells were lysed using a cold lysis buffer containing protease and phosphatase inhibitors (Santa Cruz Biotechnology, Santa Cruz, CA, USA). The lysates were centrifuged, and the supernatants were collected. Western blotting analysis was performed by denaturing $25 \mathrm{mg}$ of protein at $95^{\circ} \mathrm{C}$ for $5 \mathrm{~min}$ in a Laemmli sample buffer and 2-mercaptoethanol. Proteins were separated by SDS-PAGE and transferred onto PVDF membranes. The membranes were blocked, and incubated with antibodies against Sirt1, Sirt6 (Abcam), NLRP3 (Santa Cruz Biotechnology), and beta-actin (Cell Signaling Technology, Danvers, MA, USA). Membranes were exposed to horseradish peroxidase-conjugated secondary antibody (Santa Cruz Biotechnology). Detection was performed using enhanced chemiluminescence (GE Healthcare, Piscataway, NJ, USA). The bands were scanned with LAS 4000 imager (Fujifilm, Duesseldorf, Germany). Densitometry analysis was performed using ImageJ software (NIH, Bethesda, MD, USA).

Tumor necrosis factor (TNF)-alpha enzyme-linked immunosorbent assay (ELISA)

Cell lysates were prepared using a cold lysis buffer. And, commercially available ELISA kit was used to measure TNF-alpha (R\&D Systems, Minneapolis, MN, USA).

\section{Statistical analysis}

Statistical analysis was performed using SPSS statistics 24 (IBM Corp., Armonk, NY, USA). Data were expressed as Mean \pm SE. Differences among experimental groups were analyzed using one-way ANOVA, followed by Bonferroni's post hoc test, where $P<0.05$ was considered significant.

\section{RESULTS}

Cell viability, cell morphology, and fat accumulation

Cytotoxicity of NR was assessed by PrestoBlue ${ }^{\circledR}$ assay in AML12 mouse hepatocytes. Addition of NR did not affect cell viability (Fig. 1A). $10 \mu \mathrm{M}$ and $10 \mathrm{mM}$ of NR treatments did not alter cell morphology (Fig. 1B). The effects of NR on fat 
(A)

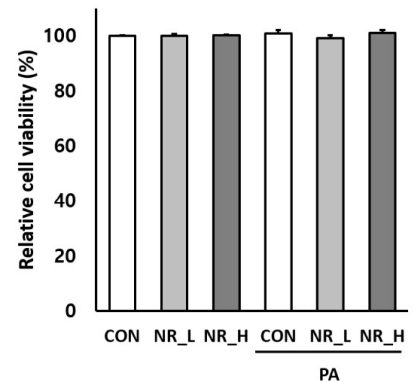

(B)

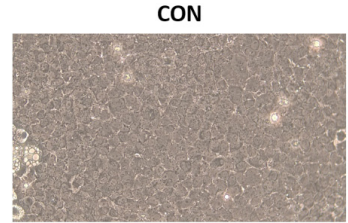

PA

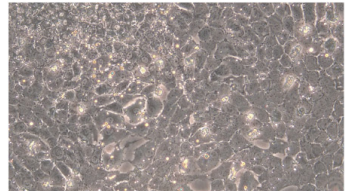

CON

(C)

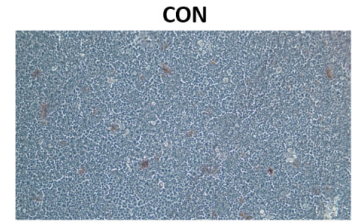

PA

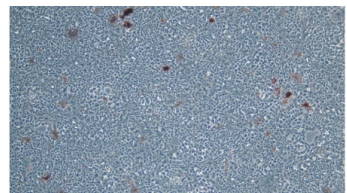

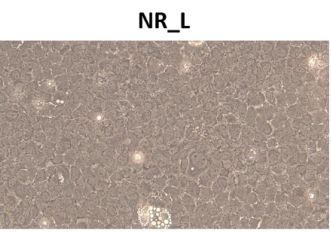

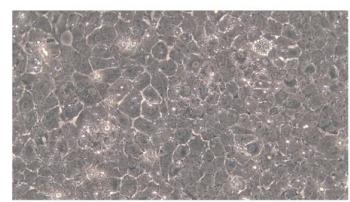

NR_L
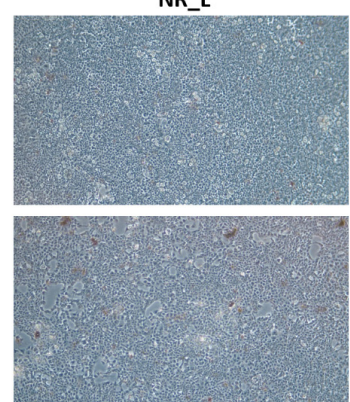
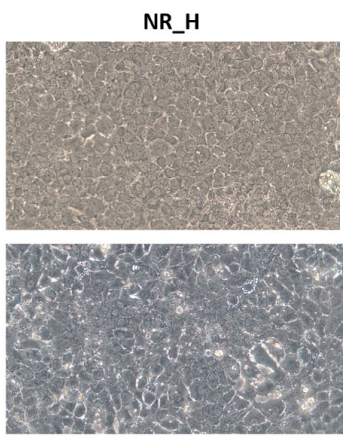

NR_H
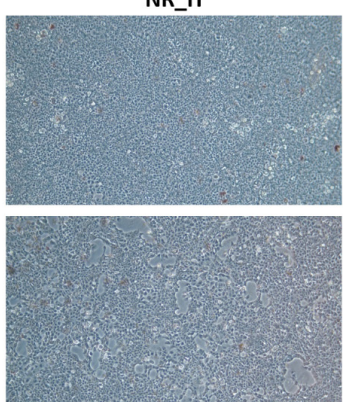

Fig. 1. Effects of nicotinamide riboside (NR) treatment on (A) cell viability, (B) cell morphology, and (C) Oil Red O staining in AML12 mouse hepatocytes. Cell viability was analyzed by PrestoBlue assay. The cells were incubated with the PrestoBlue reagent, and the absorbance was measured using a microplate reader at 570 nm. Data are expressed as Mean \pm SE. Morphological changes in the cells were captured using a microscope attached with camera (original magnification $200 \mathrm{x}$ ). (C) Representative images of Oil Red $\mathrm{O}$ staining in AML12 mouse hepatocytes (original magnification $40 \mathrm{x}$ ). NR_L, low-dose $(10 \mu \mathrm{M}) \mathrm{NR}$; NR_H, high-dose (10 mM) NR; PA, palmitic acid.

(A)

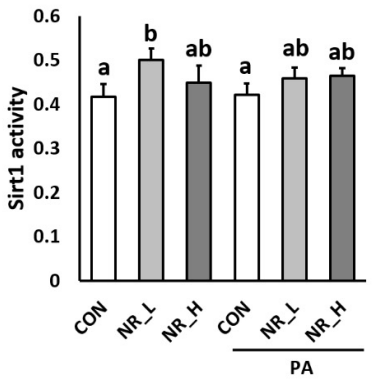

(E)

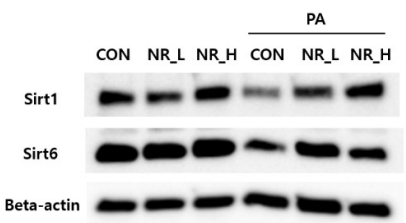

(B)

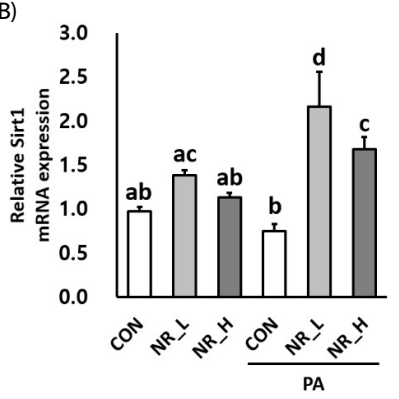

(F)

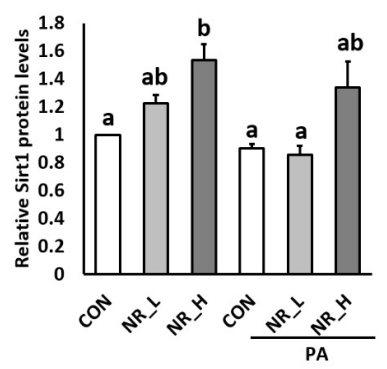

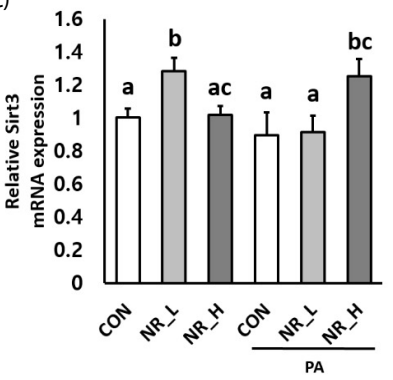

(D)

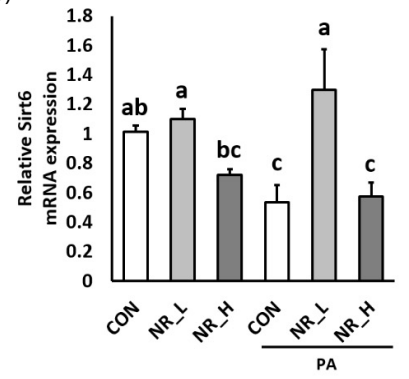

(G)

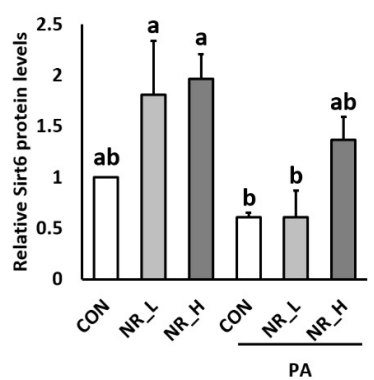

Fig. 2. Effects of nicotinamide riboside (NR) treatment on (A) sirtuin1 (Sirt1) activity, and the gene expression of Sirt1 (B), Sirt3 (C) and Sirt6 (D) in AML12 mouse hepatocytes. Gene expression of each target was normalized to that of 18S. Representative Western blots (E) and densitometric analysis for Sirt1 (F) and Sirt6 (G). Relative densitometry data of each target were normalized to those of beta-actin. Data are expressed as Mean $\pm \mathrm{SE}(n=5-6$ per group). Different letters within a variable are significantly different at $P<0.05$. NR_L, low-dose $(10 \mu \mathrm{M}) \mathrm{NR}$; NR_H, high-dose $(10 \mathrm{mM})$ NR; PA, palmitic acid.

accumulation were examined by Oil Red O staining of AML12 hepatocytes. PA treatment tended to increase fat accumulation which was stained with pink or red colors (Fig. 1C). NR treatment tended to reduce fat accumulation, as indicated by decreased Oil Red O staining in AML12 hepatocytes. 
(A)

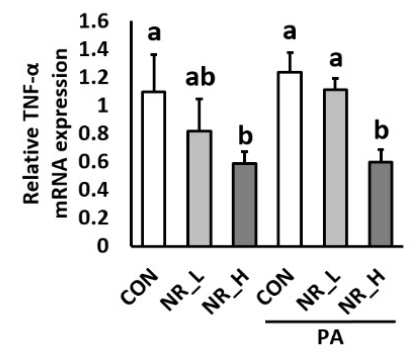

(E)

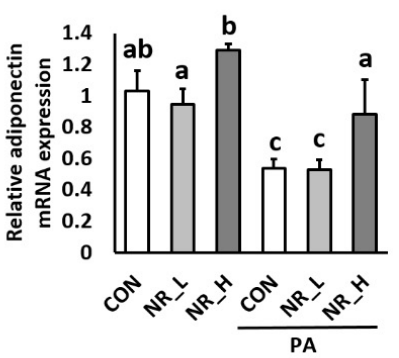

(

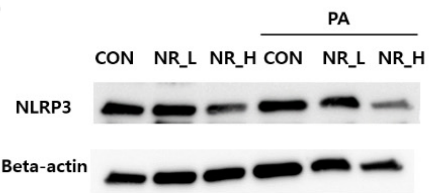

(B)

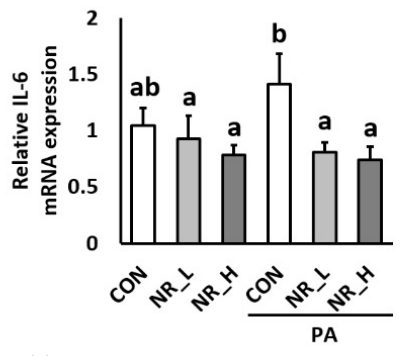

(F)

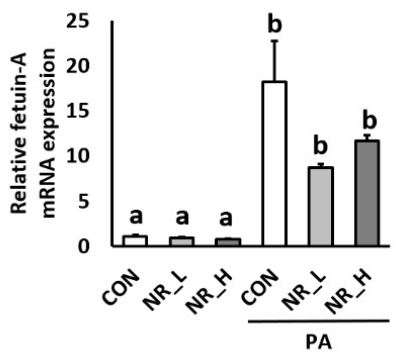

(l)

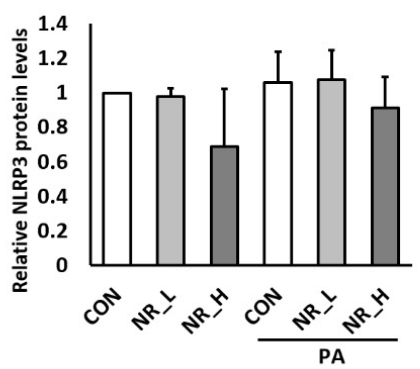

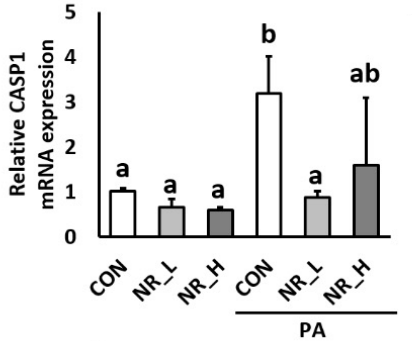

(G)

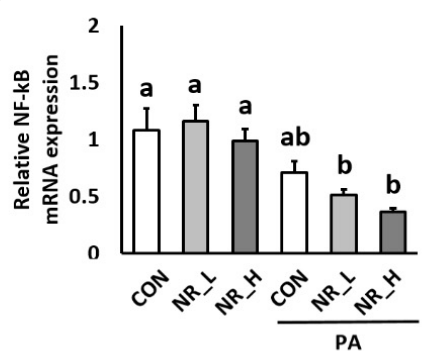

(J)

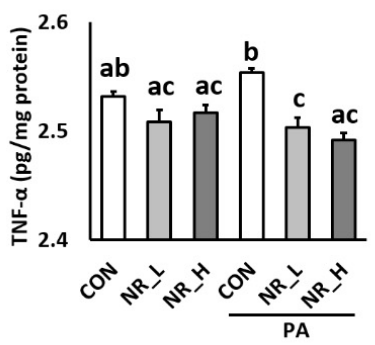

Fig. 3. Effects of NR treatment on inflammation in AML12 mouse hepatocytes. The gene expression of (A) tumor necrosis factor-alpha (TNF-a), (B) interleukin-6 (IL-6), (C) caspase1 (CASP1), (D) peroxisome proliferator-activated receptor-alpha (PPAR-a), (E) adiponectin, ( $\mathrm{F}$ ) fetuin-A, and (G) nuclear factor kappa B (NF-kB) ( $n=5-6$ per group). Gene expression of each target was normalized to that of $18 \mathrm{~S}$. (H) Representative Western blots and (I) densitometric analysis for nucleotide binding and oligomerization domain-like receptor family, pyrin domain containing 3 (NLRP3) inflammasome. Relative densitometry data of each target were normalized to those of beta-actin. (J) Protein concentrations of TNF-a in PA-untreated and -treated AML12 mouse hepatocytes analyzed by enzyme-linked immunosorbent assay. Data are expressed as Mean \pm SE. Different letters within a variable are significantly different at $P<0.05$. NR_L, low-dose $(10 \mu \mathrm{M}) \mathrm{NR}$; NR_H, high-dose $(10 \mathrm{mM}) \mathrm{NR}$; PA, palmitic acid

\section{Effects of NR on gene expression and activity of sirtuins}

To test whether NR treatment affected sirtuins, the expression levels of Sirt1, Sirt3, Sirt6 and Sirt1 activity were evaluated. Low-dose $(10 \mu \mathrm{M})$ NR significantly increased Sirt1 activity and Sirt3 expression without altering the gene expression of Sirt1 and Sirt6 in PA-untreated hepatocytes (Fig. 2). Unexpectedly, high-dose $(10 \mathrm{mM}) \mathrm{NR}$ had no effect on the gene expression and activity of sirtuins in PA-untreated hepatocytes. In a condition of hepatocyte steatosis, the increases in Sirt1 and Sirt6 expression following treatment with low-dose NR were noticeable, which was even higher than under high-dose NR (Fig. 2B and 2D). Sirt1 activity (Fig. 2A) was not affected by doubling the dose of NR treatment in PA-treated cells. Sirt3 expression was upregulated by high-dose NR, and not by low-dose NR in PA-treated hepatocytes (Fig. 2C). High-dose NR significantly increased Sirt1 protein levels in PA-untreated hepatocytes (Fig. $2 \mathrm{~F})$, however, the effects of two doses of NR on Sirt1 protein levels were not statistically significant in PA-treated hepatocytes even though the $P$-values were close to the significance levels (CON vs. NR_H, $P=0.052$ ) in PA-treated hepatocytes (Fig. 2F).
Sirt6 protein levels were not significantly altered by two doses of NR (Fig. 2G).

\section{Alteration of inflammation-related markers by $N R$}

To investigate the role of NR treatment in inflammation, the expression TNF-alpha, interleukin (IL)-6, NLRP3 inflammasome components, peroxisome proliferator-activated receptor (PPAR)alpha, adiponectin, fetuin-A, and nuclear factor kappa B (NF- $\kappa B$ ) was analyzed. High-dose NR significantly decreased TNF-alpha expression in PA-untreated and -treated hepatocytes (Fig. 3A). Two doses of NR significantly reduced protein concentration of TNF-alpha in PA-treated hepatocytes (Fig. 3J). Gene expression of IL- 6 and caspase 1 as well as NLRP3 protein levels were not affected by NR in PA-untreated hepatocytes (Fig. 3B, 3C, 3H, and $3 \mathrm{I}$ ). PA treatment induced hepatocyte steatosis, and significantly upregulated caspase 1 expression (Fig. 3C). Low-dose NR treatment significantly downregulated IL- 6 and caspase 1 in PA-treated hepatocytes (Fig. 3B and 3C). High-dose NR treatment altered IL- 6 expression, but not caspase1 in PA-treated hepatocytes (Fig. 3B and 3C). PPAR-alpha mRNA expression was 
(A)

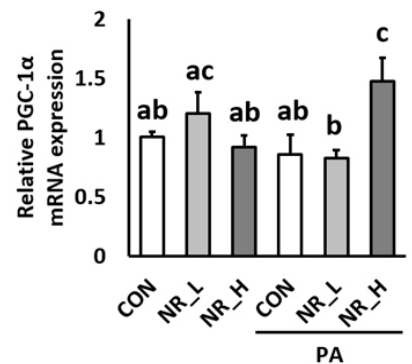

(D)

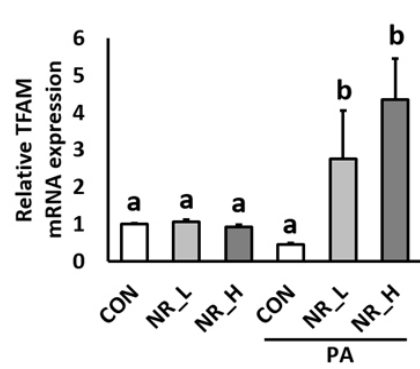

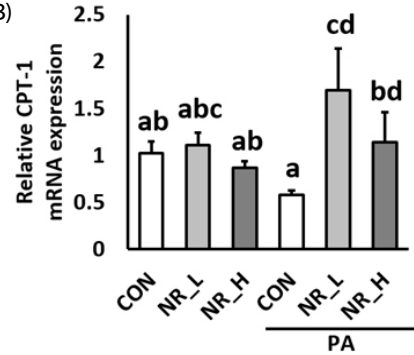

(E)

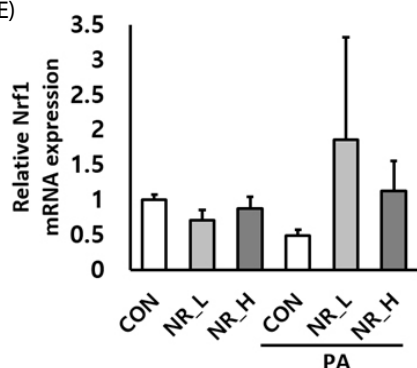

(C)
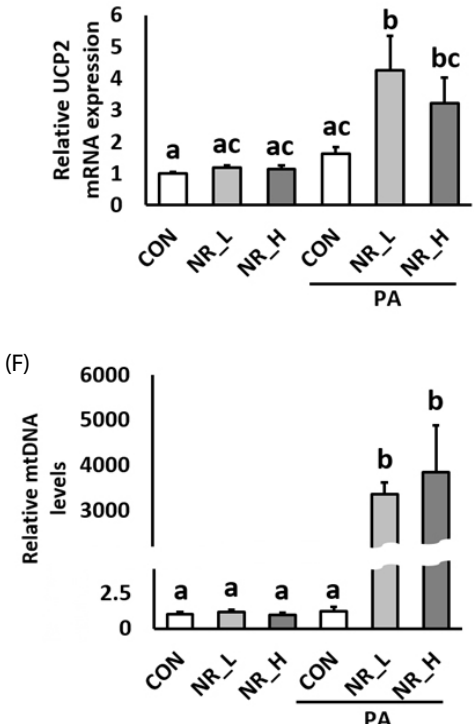

Fig. 4. Effects of NR treatment on the gene expression levels of (A) peroxisome proliferator-activated receptor gamma coactivator-1 alpha (PGC-1a), (B) carnitine palmitoyltransferase 1 (CPT-1), (C) uncoupling protein 2 (UCP2), (D) transcription factor A, mitochondrial (TFAM), (E) nuclear respiratory factor 1 (Nrf1), and (F) mitochondrial DNA (mtDNA). Gene expression of each target was normalized to that of $18 \mathrm{~S}$. Data are expressed as Mean \pm SE ( $n=5-6$ per group). Different letters within a variable are significantly different at $P<0.05$. NR_L, low-dose $(10 \mu \mathrm{M}) \mathrm{NR}$; NR_H, high-dose $(10 \mathrm{mM}) \mathrm{NR}$; PA, palmitic acid.

increased by low-dose NR treatment in PA-untreated hepatocytes (Fig. 3D). PA treatment did not affect PPAR-alpha level. Highdose NR significantly increased PPAR-alpha expression, and not by low-dose NR in PA-treated hepatocytes (Fig. 3D). Adiponectin gene expression in PA-untreated hepatocyte was not affected by NR (Fig. 3E). Induction of hepatocyte steatosis by PA treatment significantly decreased adiponectin level, which was rescued by high-dose NR treatment (Fig. 3E). Gene expression of fetuin-A and NF- $\kappa B$ was not altered by doubling the dose of NR in PA-untreated and -treated hepatocytes (Fig. 3F and 3G). PA treatment significantly upregulated fetuin-A level, and low-dose NR tended to downregulate fetuin-A levels $(P=0.057)$ (Fig. 3F).

\section{Effects of NR on mitochondria-related markers}

To determine the effect of NR on mitochondrial markers, the gene expression of peroxisome proliferator-activated receptor gamma coactivator-1 alpha (PGC-1a), carnitine palmitoyltransferase 1 (CPT-1), uncoupling protein 2 (UCP2), transcription factor $\mathrm{A}$, mitochondrial (TFAM) and nuclear respiratory factor 1 (Nrf1) as well as mitochondrial DNA (mtDNA) in AML12 hepatocytes was analyzed. NR treatment did not affect the expression of these mitochondrial markers in PA-untreated hepatocytes and Nrf1 in PA-untreated and -treated hepatocytes (Fig. 4). UCP2 gene expression was increased by low dose of NR in PA-treated hepatocytes (Fig. 4C). High-dose NR treatment significantly increased PGC-1a in PA-treated hepatocytes (Fig. $4 A)$. Low and high doses of NR treatment significantly increased CPT-1, TFAM, and mtDNA in a condition of hepatocyte steatosis (Fig. 4B, 4D and 4F).

\section{DISCUSSION}

The present study investigated whether NR attenuated hepatic inflammation and altered mitochondrial markers in mouse hepatocytes. Administration of NR did not affect cell viability or morphology. No undesirable effects of NR in animals and humans have been reported until now $[6,9,11,15]$. Further, findings from the current study supported the hypothesis that NR treatment attenuates hepatic inflammation and induces mitochondrial biogenesis in mouse hepatocytes.

NR prevented the development of HF diet-induced NAFLD in an aged mouse model [17]. In addition, NR administration attenuated hepatic metaflammation by modulating the NLRP3 inflammasome levels in a mouse model of type 2 diabetes [15]. NR delayed the progression of mitochondrial myopathy by boosting mitochondrial biogenesis and preventing mitochondrial DNA deletion in a mouse model of mitochondrial myopathy [12]. These results suggest the possibility of NR as a therapeutic agent for liver diseases by improving inflammation and mitochondrial biogenesis.

Sirtuins are a family of seven proteins that function as $\mathrm{NAD}^{+}$-dependent deacetylases. Among the seven sirtuins, which exhibit distinct functions and locations, Sirt1, 2, 3, 6, and 7 exert anti-inflammatory effects in fatty liver disease, metabolic syndrome and other chronic inflammatory diseases [18-21]. Moreover, Sirt3, mainly located in mitochondria, is involved in mitochondrial function and mitochondrial diseases [22-24]. As expected, NR treatment significantly upregulated Sirt1 gene expression. Sirt3 gene expression was significantly upregulated by $\mathrm{NR}$ in hepatocytes (Fig. $2 \mathrm{~B}$ and $2 \mathrm{C}$ ). These alterations may suggest that these sirtuins play a role in NR-mediated effects on inflammation and mitochondrial function. Despite some interesting findings on NR and sirtuins in this study, our analyses had limitations; part of interpretation from the data was based on the alteration of mRNA expression, and there was discrepancy between mRNA expression and protein levels. The discrepancy 
may be due to post-transcriptional (e.g. mRNA stability), translational (e.g. initiation factor and trans-acting protein) or posttranslational (e.g. proteolysis and phosphorylation) regulations. Transcription of Sirt was regulated by various transcription factors. For example, Forkhead box 01, PPAR-alpha/beta, and CAMP response element-binding are known to increase Sirt1 expression [25-28]; however, PPAR-gamma and carbohydrate response element-binding protein down-regulate Sirt1 expression $[26,29]$. On the other hand, post-transcriptional or translational regulation of Sirt was not well investigated. Also, Sirt activity is the most direct factor regulating deacetylase activity of sirtuins, and is regulated at various levels. Two main types of post-translational modifications of Sirt are phosphorylation and sumoylation [30-33]. Sirt can be phosphorylated by cyclin B-cyclin-dependent kinase 1 complex, JUN Nterminal kinase, dual specificity Tyr-phosphorylated and regulated kinase 1 (DYRK1) and DYRK3 [30-32]. Moreover, sumoylation of Sirt1 increases Sirt1 activity [33]. In addition to post-translational modifications, formation of complexes between Sirt and other positive (active regulator of Sirt1) and negative proteins (nuclear receptor co-repressor 1-silencing mediator of retinoid and thyroid hormone receptors complex and deleted in breast cancer 1) as well as NAD ${ }^{+}$availability can alter Sirt activity [34-38]. In our study, low dose NR increased Sirt1 activity in PAuntreated hepatocytes, but, the NR-mediated increases in Sirt1 activity were not shown in PA-treated hepatocytes (Fig. 2A). In addition to these limitations, lack of dose-dependent responses of NR on sirtuins made the clear interpretation difficult.

Inflammation plays a major role in the pathogenesis of NAFLD and obesity-induced insulin resistance [39]. A coordinated network of various pro-inflammatory and anti-inflammatory cytokines controls the inflammatory response. In the current study, NR ameliorated hepatic inflammation by reducing the levels of the pro-inflammatory cytokines, TNF-a and IL-6 (Fig. $3 \mathrm{~A}, 3 \mathrm{~B}$, and $3 \mathrm{~J})$. The inflammatory response accompanies systemic activation of many signaling pathways. NF- $\kappa B$ induces the expression and activation of inflammatory cytokines [40]. Knockdown of NLRP3 reduced NF- $\kappa B$ activation in human monocyte cell line THP-1 [41]. In the present study, NR upregulated anti-inflammatory molecule adiponectin, and tended to downregulate hepatokine fetuin-A levels (Fig. 3E and $3 F)$. High levels of fetuin-A have been reported in animal models of diet-induced obesity (DIO) and obese diabetic patients, whereas reduction of hepatic fat content is associated with the decline of fetuin-A in humans $[42,43]$. Resveratrol lowered fetuin-A in a mouse model of DIO with direct interaction of adiponectin and fetuin-A [2]. NR appears to inversely regulate adiponectin and fetuin- $A$ in mouse hepatocytes.

Accumulating evidence indicates that mitochondrial dysfunction contributes to the pathogenesis of liver diseases including NAFLD because it affects hepatic lipid homeostasis, and promotes reactive oxygen species production, lipid peroxidation, and cytokine release [44]. Impaired mitochondrial biogenesis occurs in Alzheimer's disease, cardiovascular disease, and aging [45-47]. Various transcriptional networks regulate the mitochondrial metabolism [48]. At the molecular level, several transcription factors and cofactors mediate in the activation and regulation of mitochondrial biogenesis [49]. PGC-1a is a master regulator of mitochondrial biogenesis by integrating and coordinating the activity of multiple transcription factors such as Nrf-1, and -2 , mitochondrial transcription factor A, and PPAR-alpha [50]. NR increased levels of mitochondrial biogenesis-related factors (PGC-1a, CPT-1, UCP2, and TFAM) as well as mtDNA (Fig. 4). The gene expression of PGC-1a is directly linked to mitochondrial biogenesis [51]. PGC-1a modulates Nrf1 and other mitochondrial biogenesis-related factors [52]. Fatty liver was associated with impaired activity of PGC-1a in mice [53]. Activation of PGC-1a leads to increased expression of genes related to gluconeogenesis, fatty acid oxidation, and lipid transport [54,55]. Our findings from the current study demonstrated that NR induced PGC-1a, CPT-1, UCP2, and TFAM as well as mtDNA in AML12 mouse hepatocytes.

This study suggests the potential role of NR in inflammation regulation and mitochondrial biogenesis in a model of hepatocyte steatosis. As inflammation and mitochondrial dysfunction are involved in the pathophysiology in NAFLD, our study has potential clinical and public health implications if the findings are confirmed in human clinical trials. In conclusion, our findings demonstrate that NR treatment attenuates hepatic inflammation, and induces mitochondrial biogenesis in mouse hepatocytes. These findings suggest the therapeutic value of NR in hepatic inflammation and impaired mitochondrial biogenesis.

\section{CONFLICT OF INTEREST}

The authors declare that they have no competing interests related with this study.

\section{ORCID}

Hee Jae Lee: https://orcid.org/0000-0003-4242-8788

Soo Jin Yang: https://orcid.org/0000-0001-7892-7648

\section{REFERENCES}

1. Trammell SA, Yu L, Redpath P, Migaud ME, Brenner C. Nicotinamide riboside is a major NAD+ precursor vitamin in cow milk. J Nutr 2016;146:957-63.

2. Belenky P, Racette FG, Bogan KL, McClure JM, Smith JS, Brenner C. Nicotinamide riboside promotes Sir2 silencing and extends lifespan via Nrk and Urh1/Pnp1/Meu1 pathways to NAD+. Cell 2007;129:473-84.

3. Bieganowski $P$, Brenner $C$. Discoveries of nicotinamide riboside as a nutrient and conserved NRK genes establish a Preiss-Handler independent route to NAD+ in fungi and humans. Cell 2004;117: 495-502.

4. Julius U. Niacin as antidyslipidemic drug. Can J Physiol Pharmacol 2015;93:1043-54.

5. Bogan KL, Brenner C. Nicotinic acid, nicotinamide, and nicotinamide riboside: a molecular evaluation of NAD+ precursor vitamins in human nutrition. Annu Rev Nutr 2008;28:115-30.

6. Yang SJ, Choi JM, Kim L, Park SE, Rhee EJ, Lee WY, Oh KW, Park SW, Park CY. Nicotinamide improves glucose metabolism and affects the hepatic NAD-sirtuin pathway in a rodent model of obesity and type 2 diabetes. J Nutr Biochem 2014;25:66-72.

7. Shima K, Zhu M, Kuwajima M. A role of nicotinamide-induced 
increase in pancreatic $\beta$-cell mass on blood glucose control after discontinuation of the treatment in partially pancreatectomized OLETF rats. Diabetes Res Clin Pract 1998;41:1-8.

8. Knip M, Douek IF, Moore WP, Gillmor HA, McLean AE, Bingley PJ, Gale EA; European Nicotinamide Diabetes Intervention Trial Group. Safety of high-dose nicotinamide: a review. Diabetologia 2000;43: 1337-45.

9. Trammell SA, Schmidt MS, Weidemann BJ, Redpath P, Jaksch F, Dellinger RW, Li Z, Abel ED, Migaud ME, Brenner C. Nicotinamide riboside is uniquely and orally bioavailable in mice and humans. Nat Commun 2016;7:12948.

10. Cantó C, Houtkooper RH, Pirinen E, Youn DY, Oosterveer $\mathrm{MH}$, Cen $\mathrm{Y}$, Fernandez-Marcos PJ, Yamamoto $\mathrm{H}$, Andreux PA, Cettour-Rose $P$, Gademann K, Rinsch C, Schoonjans K, Sauve AA, Auwerx J. The $\mathrm{NAD}^{+}$precursor nicotinamide riboside enhances oxidative metabolism and protects against high-fat diet-induced obesity. Cell Metab 2012;15:838-47.

11. Brown KD, Maqsood S, Huang JY, Pan Y, Harkcom W, Li W, Sauve A, Verdin E, Jaffrey SR. Activation of SIRT3 by the NAD ${ }^{+}$precursor nicotinamide riboside protects from noise-induced hearing loss. Cell Metab 2014;20:1059-68.

12. Khan NA, Auranen M, Paetau I, Pirinen E, Euro L, Forsström S, Pasila L, Velagapudi V, Carroll CJ, Auwerx J, Suomalainen A. Effective treatment of mitochondrial myopathy by nicotinamide riboside, a vitamin B3. EMBO Mol Med 2014.6:721-31.

13. Trammell SA, Weidemann BJ, Chadda A, Yorek MS, Holmes A, Coppey $\sqcup$, Obrosov A, Kardon RH, Yorek MA, Brenner C. Nicotinamide riboside opposes type 2 diabetes and neuropathy in mice. Sci Rep 2016;6:26933.

14. Gariani K, Menzies KJ, Ryu D, Wegner CJ, Wang X, Ropelle ER, Moullan N, Zhang H, Perino A, Lemos V, Kim B, Park YK, Piersigilli A, Pham TX, Yang Y, Ku CS, Koo Sl, Fomitchova A, Cantó C, Schoonjans $K$, Sauve AA, Lee JY, Auwerx J. Eliciting the mitochondrial unfolded protein response by nicotinamide adenine dinucleotide repletion reverses fatty liver disease in mice. Hepatology 2016;63:1190-204.

15. Lee HJ, Hong YS, Jun W, Yang SJ. Nicotinamide riboside ameliorates hepatic metaflammation by modulating NLRP3 inflammasome in a rodent model of type 2 diabetes. J Med Food 2015;18:1207-13.

16. Mukherjee S, Chellappa K, Moffitt A, Ndungu J, Dellinger RW, Davis JG, Agarwal B, Baur JA. Nicotinamide adenine dinucleotide biosynthesis promotes liver regeneration. Hepatology 2017;65:616-30.

17. Zhou CC, Yang X, Hua X, Liu J, Fan MB, Li GQ, Song J, Xu TY, Li ZY, Guan YF, Wang P, Miao CY. Hepatic NAD deficiency as a therapeutic target for non-alcoholic fatty liver disease in ageing. Br J Pharmacol 2016;173:2352-68.

18. Stafeev IS, Menshikov MY, Tsokolaeva ZI, Shestakova MV, Parfyonova YV. Molecular mechanisms of latent inflammation in metabolic syndrome. possible role of sirtuins and peroxisome proliferatoractivated receptor type ₹. Biochemistry (Mosc) 2015;80:1217-26.

19. Mendes KL, Lelis DF, Santos SH. Nuclear sirtuins and inflammatory signaling pathways. Cytokine Growth Factor Rev 2017;38:98-105.

20. Ding RB, Bao J, Deng CX. Emerging roles of SIRT1 in fatty liver diseases. Int J Biol Sci 2017;13:852-67.

21. Liu P, Huang G, Wei T, Gao J, Huang C, Sun M, Zhu L, Shen W. Sirtuin 3-induced macrophage autophagy in regulating NLRP3 inflammasome activation. Biochim Biophys Acta Mol Basis Dis 2018;1864:764-77.

22. Paulin R, Dromparis P, Sutendra G, Gurtu V, Zervopoulos S, Bowers
L, Haromy A, Webster L, Provencher S, Bonnet S, Michelakis ED. Sirtuin 3 deficiency is associated with inhibited mitochondrial function and pulmonary arterial hypertension in rodents and humans. Cell Metab 2014;20:827-39.

23. Gleave $J A$, Arathoon LR, Trinh $D$, Lizal $K E$, Giguère $N$, Barber $J H$, Najarali Z, Khan MH, Thiele SL, Semmen MS, Koprich JB, Brotchie JM, Eubanks JH, Trudeau LE, Nash JE. Sirtuin 3 rescues neurons through the stabilisation of mitochondrial biogenetics in the virally-expressing mutant a-synuclein rat model of parkinsonism. Neurobiol Dis 2017;106:133-46.

24. Li Y, Ye Z, Lai W, Rao J, Huang W, Zhang X, Yao Z, Lou T. Activation of sirtuin 3 by silybin attenuates mitochondrial dysfunction in cisplatin-induced acute kidney injury. Front Pharmacol 2017;8:178.

25. Nemoto S, Fergusson MM, Finkel T. Nutrient availability regulates SIRT1 through a forkhead-dependent pathway. Science 2004;306: 2105-8.

26. Noriega LG, Feige JN, Canto C, Yamamoto H, Yu J, Herman MA, Mataki C, Kahn BB, Auwerx J. CREB and ChREBP oppositely regulate SIRT1 expression in response to energy availability. EMBO Rep 2011;12:1069-76.

27. Hayashida S, Arimoto A, Kuramoto Y, Kozako T, Honda S, Shimeno $\mathrm{H}$, Soeda $\mathrm{S}$. Fasting promotes the expression of SIRT1, an NAD+dependent protein deacetylase, via activation of PPARa in mice. Mol Cell Biochem 2010;339:285-92.

28. Okazaki M, Iwasaki Y, Nishiyama M, Taguchi T, Tsugita M, Nakayama S, Kambayashi M, Hashimoto K, Terada Y. PPAR $/ \delta$ regulates the human SIRT1 gene transcription via Sp1. Endocr J 2010;57:403-13.

29. Han L, Zhou R, Niu J, McNutt MA, Wang P, Tong T. SIRT1 is regulated by a PPARY-SIRT1 negative feedback loop associated with senescence. Nucleic Acids Res 2010;38:7458-71.

30. Sasaki T, Maier B, Koclega KD, Chruszcz M, Gluba W, Stukenberg PT, Minor W, Scrable H. Phosphorylation regulates SIRT1 function. PLoS One 2008;3:e4020.

31. Nasrin N, Kaushik VK, Fortier E, Wall D, Pearson KJ, de Cabo R, Bordone L. JNK1 phosphorylates SIRT1 and promotes its enzymatic activity. PLoS One 2009;4:e8414.

32. Guo X, Williams JG, Schug TT, Li X. DYRK1A and DYRK3 promote cell survival through phosphorylation and activation of SIRT1. J Biol Chem 2010;285:13223-32.

33. Yang Y, Fu W, Chen J, Olashaw N, Zhang X, Nicosia SV, Bhalla K, Bai W. SIRT1 sumoylation regulates its deacetylase activity and cellular response to genotoxic stress. Nat Cell Biol 2007;9:1253-62.

34. Kim EJ, Kho JH, Kang MR, Um SJ. Active regulator of SIRT1 cooperates with SIRT1 and facilitates suppression of p53 activity. Mol Cell 2007;28:277-90.

35. Picard F, Kurtev M, Chung N, Topark-Ngarm A, Senawong T, Machado De Oliveira R, Leid M, McBurney MW, Guarente L. Sirt1 promotes fat mobilization in white adipocytes by repressing PPAR-X. Nature 2004;429:771-6.

36. Kim JE, Chen J, Lou Z. DBC1 is a negative regulator of SIRT1. Nature 2008;451:583-6.

37. Zhao W, Kruse JP, Tang Y, Jung SY, Qin J, Gu W. Negative regulation of the deacetylase SIRT1 by DBC1. Nature 2008;451:587-90.

38. Houtkooper RH, Pirinen E, Auwerx J. Sirtuins as regulators of metabolism and healthspan. Nat Rev Mol Cell Biol 2012;13:225-38.

39. Cohen JC, Horton JD, Hobbs HH. Human fatty liver disease: old questions and new insights. Science 2011;332:1519-23.

40. Kinoshita T, Imamura R, Kushiyama H, Suda T. NLRP3 mediates NF- $\kappa$ 
B activation and cytokine induction in microbially induced and sterile inflammation. PLoS One 2015;10:e0119179.

41. Tak PP, Firestein GS. NF-kB: a key role in inflammatory diseases. J Clin Invest 2001;107:7-11.

42. Lin X, Braymer HD, Bray GA, York DA. Differential expression of insulin receptor tyrosine kinase inhibitor (fetuin) gene in a model of diet-induced obesity. Life Sci 1998;63:145-53.

43. Lee HJ, Lim Y, Yang SJ. Involvement of resveratrol in crosstalk between adipokine adiponectin and hepatokine fetuin- $\mathrm{A}$ in vivo and in vitro. J Nutr Biochem 2015;26:1254-60.

44. Begriche K, Igoudjil A, Pessayre D, Fromenty B. Mitochondrial dysfunction in NASH: causes, consequences and possible means to prevent it. Mitochondrion 2006;6:1-28.

45. Sheng B, Wang X, Su B, Lee HG, Casadesus G, Perry G, Zhu X. Impaired mitochondrial biogenesis contributes to mitochondrial dysfunction in Alzheimer's disease. J Neurochem 2012;120:419-29.

46. Ren J, Pulakat L, Whaley-Connell A, Sowers JR. Mitochondrial biogenesis in the metabolic syndrome and cardiovascular disease. J Mol Med (Berl) 2010;88:993-1001.

47. López-Lluch G, Irusta PM, Navas P, de Cabo R. Mitochondrial biogenesis and healthy aging. Exp Gerontol 2008;43:813-9.

48. Austin S, St-Pierre J. PGC1a and mitochondrial metabolism--emerging concepts and relevance in ageing and neurodegenerative disorders. J Cell Sci 2012;125:4963-71.

49. Goffart S, Wiesner RJ. Regulation and co-ordination of nuclear gene expression during mitochondrial biogenesis. Exp Physiol 2003;88: 33-40.

50. Handschin C, Spiegelman BM. Peroxisome proliferator-activated receptor gamma coactivator 1 coactivators, energy homeostasis, and metabolism. Endocr Rev 2006;27:728-35.

51. Gong B, Pan Y, Vempati P, Zhao W, Knable L, Ho L, Wang J, Sastre $M$, Ono K, Sauve AA, Pasinetti GM. Nicotinamide riboside restores cognition through an upregulation of proliferator-activated receptor- $\gamma$ coactivator 1 a regulated $\beta$-secretase 1 degradation and mitochondrial gene expression in Alzheimer's mouse models. Neurobiol Aging 2013;34:1581-8.

52. Fernandez-Marcos PJ, Auwerx J. Regulation of PGC-1a, a nodal regulator of mitochondrial biogenesis. Am J Clin Nutr 2011;93: 884S-890S.

53. Aharoni-Simon M, Hann-Obercyger M, Pen S, Madar Z, Tirosh $O$. Fatty liver is associated with impaired activity of PPARY-coactivator 1a (PGC1a) and mitochondrial biogenesis in mice. Lab Invest 2011;91:1018-28.

54. Finck BN, Kelly DP. PGC-1 coactivators: inducible regulators of energy metabolism in health and disease. J Clin Invest 2006;116: 615-22.

55. Puigserver P. Tissue-specific regulation of metabolic pathways through the transcriptional coactivator PGC1-a. Int J Obes (Lond) 2005;29 Suppl 1:S5-9. 